\title{
Taylor series expansion in discrete Clifford analysis
}

\author{
Hilde De Ridder* Hennie De Schepper ${ }^{\dagger} \quad$ Frank Sommen ${ }^{\ddagger}$
}

\begin{abstract}
Discrete Clifford analysis is a discrete higher-dimensional function theory which corresponds simultaneously to a refinement of discrete harmonic analysis and to a discrete counterpart of Euclidean Clifford analysis. The discrete framework is based on a discrete Dirac operator that combines both forward and backward difference operators and on the splitting of the basis elements $\mathbf{e}_{j}=\mathbf{e}_{j}^{+}+\mathbf{e}_{j}^{-}$into forward and backward basis elements $\mathbf{e}_{j}^{ \pm}$. For a systematic development of this function theory, an indispensable tool is the Taylor series expansion, which decomposes a discrete (monogenic) function in terms of discrete homogeneous (monogenic) building blocks. The latter are the so-called discrete Fueter polynomials. For a discrete function, the authors assumed a series expansion which is formally equivalent to the Taylor series expansion in Euclidean Clifford analysis; however, attention needed to be paid to the geometrical conditions on the domain of the function, the convergence and the equivalence to the given discrete function. We furthermore applied the theory to discrete delta functions and investigated the connection with Shannon sampling theorem (C.E. Shannon, A mathematical theory of communication, 1948).

We found that any discrete functions admits a series expansion into discrete homogeneous polynomials and any discrete monogenic function admits a Taylor series expansion in terms of the discrete Fueter polynomials, i.e. discrete homogeneous monogenic polynomials. Although formally the discrete Taylor series expansion of a function resembles the continuous Taylor series expansion, the main difference is that there is no restriction on discrete functions to be represented as infinite series of discrete homogeneous polynomials. Finally, since the continuous expansion of the Taylor series expansion of discrete delta functions is a sinc function, the discrete Taylor series expansion lays a link with Shannon sampling.
\end{abstract}

MSC Classification: (primary) 39A12, 41A58, 44A55 (secondary) 12H10, 15A66, 39A70

Key Words: Taylor series expansion, discrete Clifford analysis, Fueter polynomials, sampling theorems

\section{Introduction}

In a recent set of papers (see a.o. $[3,5,6,7]$ ), we introduced a function theory within discrete Clifford analysis, i.e. the theory of null solutions of a discrete Dirac operator, called discrete monogenic functions. Unlike some other recent approaches towards discrete Clifford analysis, which mainly started from the desired application to potential theory, see [2], we have chosen to introduce first a general theoretical framework for our discrete function theory, more precisely, in a Hermitian setting $([3,4,10])$. Fundamental to this approach is the splitting of the basis vectors $\mathbf{e}_{j}$ into a forward and backward part $\mathbf{e}_{j}^{ \pm}$, in order to combine them with the corresponding forward and backward difference operators, enabling us to introduce a Dirac operator $D$ which factorizes the star

\footnotetext{
*Clifford Research Group, Faculty of Engineering, Ghent University, Belgium, hdr@cage.ugent.be

${ }^{\dagger}$ Clifford Research Group, Faculty of Engineering, Ghent University, Belgium, hds@cage.ugent.be

${ }^{\ddagger}$ Clifford Research Group, Faculty of Sciences, Ghent University, Belgium, fs@cage.ugent.be
} 
Laplacian $\left(D^{2}=\Delta_{h}^{*}\right)$. This distinguishes our approach also from other theoretical contributions to the field, where only one type of difference operator was used, see [8].

In [5] a discrete Fischer decomposition, the related monogenic projection and the dimension of the spaces of discrete monogenic and discrete homogeneous polynomials were established. In [6], we developed a discrete Cauchy-Kovalevskaya extension theorem which we used to introduce a basis for the space of discrete spherical monogenics, i.e. discrete homogeneous monogenic polynomials, the so-called Fueter polynomials, the properties of which are explicitely described in [7].

In this paper, we continue the development of the discrete monogenic function theory with an indispensable tool in the function theoretical toolbox: the Taylor series expansions of discrete functions. In Euclidean Clifford analysis, monogenic functions can be expressed in an infinite series expansion in terms of the so-called Fueter polynomials, i.e. discrete homogeneous solutions to the Dirac operator. In this contribution, we will show that an equivalent property holds for discrete functions: any discrete monogenic function can be expanded in an infinite series, consisting of discrete monogenic homogeneous polynomials, the so-called discrete Fueter polynomials.

Theorem 1.1. Let $\Omega$ be a bounded set as in Lemma 3.3 and let the function $f$ be defined on the set (12). If $f$ is left discrete monogenic in $\Omega$, then $f$ can be developed into a convergent series of spherical monogenics as follows:

$$
f(x)=\sum_{k=0}^{\infty}\left(\sum_{\left(\ell_{1}, \ldots, \ell_{k}\right)} V_{\ell_{1}, \ldots, \ell_{k}} \partial_{\ell_{k}} \ldots \partial_{\ell_{1}} f(0)\right)
$$

Moreover, any derived series (whenever it is defined) is normally convergent in $\Omega$ to the corresponding 'derivative' of $f$, i.e., if $j$ denotes a single summation index, the terms of the multiple power series being ordered in an arbitrary way, then for each bounded $K \subset \Omega$ and each multi-index $\underline{\beta}=\left(\beta_{1}, \ldots, \beta_{m}\right) \in \mathbb{N}^{m}$, it holds that

$$
\sum_{j=J}^{J^{\prime}} \sup _{x \in K}\left(\partial_{1}^{\beta_{1}} \ldots \partial_{m}^{\beta_{m}}\left(V_{\ell_{1}, \ldots, \ell_{k}} \partial_{\ell_{k}} \ldots \partial_{\ell_{1}} f(0)\right)\right) \rightarrow 0 \text { if } \inf \left(J, J^{\prime}\right) \rightarrow \infty
$$

Furthermore, even discrete functions that are not monogenic can be expressed in an infinite series now consisting of discrete homogeneous polynomials, without restrictions for the convergence. In particular, a discrete function $f$, defined on $\mathbb{Z}_{h}^{m}$ can be expressed as

$$
f(x):=\sum_{k=0}^{\infty} \frac{1}{k !} \sum_{\ell_{1}=1}^{m} \ldots \sum_{\ell_{k}=1}^{m} \xi_{\ell_{1}} \ldots \xi_{\ell_{k}}[1](x) \partial_{\ell_{k}} \ldots \partial_{\ell_{1}} f(0)
$$

This is an important result, since it allows us to expand the domain of operators which are defined only on the discrete homogeneous polynomials $\xi_{\ell_{1}} \ldots \xi_{\ell_{k}}[1]$ to all discrete functions.

The main task in the development of the discrete Taylor series expansion was proving the equivalence of the proposed Taylor series expansion to the given discrete function. Therefore, we examined under which conditions the given function is actually equivalent to its Taylor series expansion on the whole set. We find that if "enough" finite differences of two discrete functions are equal, the two functions will be the same on a given set, or equivalently, if "enough" finite differences of a discrete function are zero, the discrete function must be zero on the given set, as expressed in the main result of section 5 : 
Theorem 1.2. Let $x=\left(x_{1}, \ldots, x_{m}\right)$ be an arbitrary, but fixed point. If it holds that

$$
\partial_{1}^{\alpha_{1}} \ldots \partial_{m}^{\alpha_{m}} f(0)=0, \quad \text { for all } \alpha_{i} \leqslant 2 \frac{\left|x_{i}\right|}{h}, i=1, \ldots, m
$$

then the discrete function $f$ is zero on the whole rectangle $\left\{y:\left|y_{i}\right| \leqslant\left|x_{i}\right|, i=1, \ldots, m\right\}$.

To keep the article self-contained, we start with some preliminaries on the discrete setting in Section 2. Formally, the discrete Taylor series expansion can be derived quite directly from the one in the continuous setting, although attention has to be paid to the resulting geometrical conditions on the domain of the function, as described in Section 3. Next, in Section 4, we consider the convergence properties of the discrete Taylor series expansion. In Section 5 we describe under which conditions the given function is actually equivalent to the Taylor series expansion on the whole set. Finally, in the last section, we apply the theory of the discrete Taylor series expansion to the discrete delta function in one dimension and we study the link with Shannon sampling.

\section{Preliminaries}

Let $\mathbb{R}^{m}$ be the $m$-dimensional Euclidean space, with orthonormal basis $\mathbf{e}_{j}, j=1, \ldots, m$. In the discrete Clifford setting we consider over this space a uniform lattice with arbitrary mesh width $h>0$, defined by

$$
\mathbb{Z}_{h}^{m}=\left\{\left(\ell_{1} h, \ell_{2} h, \ldots, \ell_{m} h\right) \mid\left(\ell_{1}, \ell_{2}, \ldots, \ell_{m}\right) \in \mathbb{Z}^{m}\right\}
$$

So a Clifford vector $\underline{x}$ will only be allowed to show co-ordinates which are integer multiples of the mesh width $h$. We then introduce the traditional one-sided forward and backward difference operators $\Delta_{j}^{ \pm}, j=1, \ldots, m$, respectively acting on a function $f$ as

$$
\Delta_{j}^{+}[f](\underline{x})=\frac{f\left(\underline{x}+h \mathbf{e}_{j}\right)-f(\underline{x})}{h}, \quad \Delta_{j}^{-}[f](\underline{x})=\frac{f(\underline{x})-f\left(\underline{x}-h \mathbf{e}_{j}\right)}{h}
$$

With respect to the $\mathbb{Z}_{h}^{m}$-neighbourhood of $\underline{x}$, the usual definition of the discrete Laplacian then explicitly reads

$$
\Delta_{h}^{*}[f](\underline{x})=\sum_{j=1}^{m} \frac{f\left(\underline{x}+h \mathbf{e}_{j}\right)-f\left(\underline{x}-h \mathbf{e}_{j}\right)}{h^{2}}-2 m \frac{f(\underline{x})}{h^{2}}=\sum_{j=1}^{m} \Delta_{j}^{+} \Delta_{j}^{-}[f](\underline{x})
$$

the notation $\Delta_{h}^{*}$ referring to this operator being called the "star Laplacian", since it contains function values at the midpoints of the faces of the cube centered at $\underline{x}$.

The discrete Dirac operator factorizing this star Laplacian is introduced using the so-called Hermitean setting. In this setting each basis element $\mathbf{e}_{j}$ is split into two basis elements $\mathbf{e}_{j}^{+}$and $\mathbf{e}_{j}^{-}$, cf. $[3,4,10]$, and we consider the free algebra over $\left\{\mathbf{e}_{j}^{+}, \mathbf{e}_{j}^{-}\right\}$, satisfying the following relations:

$$
\mathbf{e}_{j}^{-} \mathbf{e}_{\ell}^{-}+\mathbf{e}_{\ell}^{-} \mathbf{e}_{j}^{-}=0=\mathbf{e}_{j}^{+} \mathbf{e}_{\ell}^{+}+\mathbf{e}_{\ell}^{+} \mathbf{e}_{j}^{+} \text {and } \mathbf{e}_{j}^{+} \mathbf{e}_{\ell}^{-}+\mathbf{e}_{\ell}^{-} \mathbf{e}_{j}^{+}=\delta_{j \ell}
$$

Since $\mathbf{e}_{j}=\mathbf{e}_{j}^{+}+\mathbf{e}_{j}^{-}$, these conditions imply that $\mathbf{e}_{j}^{2}=+1, j=1, \ldots, m$, i.e. the Clifford algebra has signature $(n, 0)$. To introduce the Dirac operator, one then combines each difference operator, forward or backward, with the corresponding forward or backward basis vector:

$$
D=\sum_{j=1}^{m} \mathbf{e}_{j}^{+} \Delta_{j}^{+}+\mathbf{e}_{j}^{-} \Delta_{j}^{-}
$$

Observe that indeed $D^{2}=\Delta_{h}^{*}$, and that $D \rightarrow \partial_{\underline{x}}$ as $h \rightarrow 0$ (for a continuous setting with the choice $\mathbf{e}_{j}^{2}=+1$, though). 
Remark 2.1. It is also possible to factorize the Laplacian using central differences by considering the Dirac operator $D=\sum_{j} e_{j} \Delta_{j}$ with $\Delta_{j} f(\underline{x})=f\left(\underline{x}+e_{j}\right)-f\left(\underline{x}-e_{j}\right)$. However, then the Laplacian is given by

$$
\Delta f(\underline{x})=\sum_{j=1}^{m}\left(f\left(\underline{x}+2 e_{j}\right)+f\left(\underline{x}-2 e_{j}\right)-2 f(\underline{x})\right)
$$

which only uses the even translations, and hence it is not so refined as when using the split $e_{j}=$ $\mathbf{e}_{j}^{+}+\mathbf{e}_{j}^{-}$. Another way out of this (see e.g. [9]) is by using half-integers $\mathbb{Z}+\frac{1}{2}=\left\{n+\frac{1}{2} \mid n \in \mathbb{Z}\right\}$, which leads to a discrete theory in which $2^{m}$ grids are being used.

Considering the (commuting) difference operators $\Delta_{j}^{ \pm}$as lowering operators, we introduce, see [5], the corresponding raising operators $X_{j}^{ \pm}(j=1, \ldots, m)$ satisfying the "skew" Weyl relations:

$$
\Delta_{j}^{+} X_{j}^{+}-X_{j}^{-} \Delta_{j}^{-}=1=\Delta_{j}^{-} X_{j}^{-}-X_{j}^{+} \Delta_{j}^{+}
$$

which replace the classical Weyl relations holding in the continuous case for the partial derivatives and the vector variable. The operators $X_{j}^{ \pm}$mutually commute and they commute also with $\Delta_{k}^{ \pm}$ for $j \neq k$.

The traditional vector variable corresponding to the Dirac operator then is replaced by the operator

$$
X=\sum_{j=1}^{m} \mathbf{e}_{j}^{+} X_{j}^{-}+\mathbf{e}_{j}^{-} X_{j}^{+}
$$

of which the components $X_{j}^{ \pm}$are no longer independent, but interconnected by (3). Next, the discrete Euler operator $E$, see also [5], is defined by imposing the intertwining relation $D X+X D=$ $2 E+m$ which holds for the Dirac operator and the vector variable in the continuous Clifford case. This discrete Euler operator $E$ is then easily seen to satisfy also the other usual intertwining relations with the Dirac operator and the vector variable respectively, i.e. $D E=E D+D$ and $E X=X E+X$. The notion of homogeneity of a discrete polynomial is then defined as follows.

Definition 2.1. A discrete polynomial $P$ is called discrete homogeneous of degree $k$ if and only if it is an eigenfunction with eigenvalue $k$ of the discrete Euler operator: $E P=k P$.

Introduction of the co-ordinate variable $\xi_{j}=X_{j}^{+} \mathbf{e}_{j}^{-}+X_{j}^{-} \mathbf{e}_{j}^{+}, j=1, \ldots, m$ and of the co-ordinate difference operator $\partial_{j}=\mathbf{e}_{j}^{+} \Delta_{j}^{+}+\mathbf{e}_{j}^{-} \Delta_{j}^{-}, j=1, \ldots, m$ enables us to decompose the discrete Dirac operator and the vector variable respectively as $D=\sum_{j=1}^{m} \partial_{j}$ and $X=\sum_{j=1}^{m} \xi_{j}$. On account of the skew Weyl relations (3), it is easily seen that $\xi_{j}$ and $\partial_{j}$ satisfy the graded (anti-)commuting relations

$$
\partial_{j} \xi_{j}-\xi_{j} \partial_{j}=1, j=1, \ldots, m, \quad \text { and } \quad \partial_{\ell} \xi_{j}+\xi_{j} \partial_{\ell}=0, \ell \neq j, j, l=1, \ldots, m
$$

However, the $\xi_{j}$ are mutually anti-commuting $\left(\xi_{j} \xi_{k}=-\xi_{k} \xi_{j}\right)$ as are the difference operators $\left(\partial_{j} \partial_{k}=-\partial_{k} \partial_{j}\right.$ for $\left.j \neq k\right)$.

Moreover, using the intertwining relation $E X=X(E+1)$, it directly follows that $E \xi_{j}=\xi_{j}(E+1)$, whence $\xi_{j}^{k}[1]$, i.e. natural powers of the operator $\xi_{j}$ acting on the ground state 1 , are the basic discrete homogeneous polynomials of degree $k$ in the variable $x_{j}$, similar to the basic homogeneous powers $x_{j}^{k}$ in the continuous setting. A closed form for these polynomials was obtained in [6]; they 
are given by

$$
\begin{aligned}
\xi_{j}^{2 n+1}[1]\left(x_{j}\right) & =x_{j}\left(\mathbf{e}_{j}^{+}+\mathbf{e}_{j}^{-}\right) \prod_{i=1}^{n}\left(x_{j}^{2}-i^{2} h^{2}\right) \\
\xi_{j}^{2 n}[1]\left(x_{j}\right) & =\left(x_{j}^{2}+n h x_{j}\left(\mathbf{e}_{j}^{+} \mathbf{e}_{j}^{-}-\mathbf{e}_{j}^{-} \mathbf{e}_{j}^{+}\right)\right) \prod_{i=1}^{n-1}\left(x_{j}^{2}-i^{2} h^{2}\right)
\end{aligned}
$$

for each $n=1,2, \ldots$ and $j=1, \ldots, m$.

Remark 2.2. Note that a 'discrete homogeneous polynomials' is not homogeneous in the classical sense, since the property $\xi_{j}^{k}[1]\left(t x_{j}\right)=t^{k} \xi_{j}^{k}[1]\left(x_{j}\right)$ does not hold.

A function defined on $\mathbb{Z}_{h}^{m}$ is then called (left) discrete monogenic in a domain $\Omega \subset \mathbb{Z}_{h}^{m}$ iff it satisfies in $\Omega$ the equation $D f=0$, or, in other words, if it is a null solution for the left action of the discrete Dirac operator. Discrete homogeneous polynomial null solutions of $D$ are called discrete spherical monogenics.

The Cauchy-Kovalevskaya extension theorem offers a tool to uniquely extend any discrete function, defined in the hyperplane $x_{1}=0$, in a monogenic way to the whole space $\mathbb{Z}_{h}^{m}$. The exact result reads as follows.

Theorem 2.1. Let the discrete function $f\left(x_{2}, \ldots, x_{m}\right)$ be defined on the grid $\mathbb{Z}_{h}^{m-1}$, and put $f_{0}=f$ and $f_{k+1}=(-1)^{k+1} D^{\prime} f_{k}(k=0,1, \ldots)$, with $D^{\prime}$ the restriction of the Dirac operator to the hyperplane $x_{1}=0$. Then

$$
C K[f]\left(x_{1}, x_{2}, \ldots, x_{m}\right)=\sum_{k=0}^{\infty} \frac{\xi_{1}^{k}[1]\left(x_{1}\right)}{k !} f_{k}\left(x_{2}, \ldots, x_{m}\right)
$$

called the Cauchy-Kovalevskaya (CK) extension of $f$, is the unique discrete monogenic function on $\mathbb{Z}_{h}^{m}$, for which the restriction to $\mathbb{Z}_{h}^{m-1}$ equals $f$.

The discrete CK extension procedure establishes an isomorphism between the space $\Pi_{k}^{(m-1)}$ of discrete homogeneous polynomials of degree $k$ in $m-1$ variables and the space $\mathcal{M}_{k}^{(m)}$ of discrete spherical monogenics of degree $k$ in $m$ variables, allowing us to determine a basis for the space $\mathcal{M}_{k}^{(m)}$.

Definition 2.2. Let $\underline{\alpha}=\left(\alpha_{2}, \ldots, \alpha_{m}\right) \in \mathbb{N}^{m-1}$ with $\alpha_{2}+\ldots+\alpha_{m}=k$. Then the discrete spherical monogenics $\bar{V}_{\underline{\alpha}}=C K\left[\xi_{2}^{\alpha_{2}} \ldots \xi_{m}^{\alpha_{m}}\right]$ are called the discrete Fueter polynomials of degree $k$. They constitute a basis for $\mathcal{M}_{k}^{(m)}$.

In what follows we will use an alternative notation for the Fueter polynomials, which is introduced as follows. Let, as in the above definition, $\underline{\alpha}=\left(\alpha_{2}, \ldots, \alpha_{m}\right) \in \mathbb{N}^{m-1}$, then we identify with $\underline{\alpha}$ the $k$-tuple $\left(\ell_{1}, \ldots, \ell_{k}\right)$, with $k=\alpha_{2}+\ldots+\alpha_{m}$. Here, the integers $\ell_{j} \in\{2, \ldots, m\}$ are such that $\ell_{j_{1}} \leq \ell_{j_{2}}$ when $j_{1}<j_{2}$ and the number of times that $i$ appears in $\left(\ell_{1}, \ldots, \ell_{k}\right)$ equals $\alpha_{i}$. We will then denote the Fueter polynomial $V_{\underline{\alpha}}$ also by $V_{\ell_{1}, \ldots, \ell_{k}}$.

\section{Taylor series expansion of $f$ in the origin}

Assume that the discrete function $f$ is defined in a bounded set $\Omega \subset \mathbb{Z}_{h}^{m}$. Without loss of generality it may be supposed that $\Omega$ contains the origin. In this section, we examine if we can write $f$ in 
the following form:

$$
f(x)=\sum_{k=0}^{\infty} \frac{1}{k !} \sum_{\ell_{1}=1}^{m} \ldots \sum_{\ell_{k}=1}^{m} \xi_{\ell_{1}} \ldots \xi_{\ell_{k}}[1](x) \lambda_{\ell_{1}, \ldots, \ell_{k}}
$$

Letting act the multi-index finite difference operator $\partial_{\ell_{k}} \ldots \partial_{\ell_{1}}$ on both sides of this equation and evaluating the result in the origin directly reveals that $\lambda_{\ell_{1}, \ldots, \ell_{k}}$ should equal $\left(\partial_{\ell_{k}} \ldots \partial_{\ell_{1}} f\right)(0)$. We will thus consider the series expansion

$$
g(x):=\sum_{k=0}^{\infty} \frac{1}{k !} \sum_{\ell_{1}=1}^{m} \ldots \sum_{\ell_{k}=1}^{m} \xi_{\ell_{1}} \ldots \xi_{\ell_{k}}[1](x) \partial_{\ell_{k}} \ldots \partial_{\ell_{1}} f(0)
$$

which indeed, at least formally, can be seen as a Taylor series expansion of $f$, when interpreting $\xi_{\ell_{1}} \ldots \xi_{\ell_{k}}[1](x)$ as the discrete counterparts of the continuous variables $x_{\ell_{1}} \ldots x_{\ell_{k}}$, and the expressions $\partial_{\ell_{k}} \ldots \partial_{\ell_{1}} f$ as the discrete counterparts of the corresponding partial derivatives. In what follows we will determine under which conditions on $f$ and on the domain $\Omega$ the series (7) can not only be formally written down, but will converge for all $x \in \Omega$ and, most importantly, will be equivalent to the original function $f(x)$ on $\Omega$.

\subsection{Domain of $f$}

In the discrete setting, partial derivatives are replaced by (forward or backward) difference operators, which implies that, theoretically speaking, in order to determine all finite differences $\partial_{\ell_{k}} \ldots \partial_{\ell_{1}} f(0)$ with $k \geqslant 0$, the function needs to be defined on the whole of $\mathbb{Z}_{h}^{m}$. However, from a pragmatic point of view, we can reduce the domain on which $f$ needs to defined. Indeed, observe from the explicit expressions (4)-(5) that, for any point $x$ in $\Omega$

$$
\xi_{j}^{r}[1]\left(x_{j}\right)=0, \quad \forall r \geqslant 2 \frac{\left|x_{j}\right|}{h}+1
$$

whence it suffices to explicitly determine only those terms $\partial_{\ell_{k}} \ldots \partial_{\ell_{1}} f(0)= \pm \partial_{1}^{\alpha_{1}} \ldots \partial_{m}^{\alpha_{m}} f(0)$ for which

$$
\alpha_{j}<2 \frac{\left|x_{j}\right|}{h}+1, j=1, \ldots, m
$$

Clearly, this observation imposes some conditions on the domain of the given function $f$, which will be further explicited below.

\subsubsection{Conditions on the domain for a single finite difference}

We first determine the necessary points required to calculate one single finite difference of the form $\partial_{\ell_{1}} \ldots \partial_{\ell_{k}} f(a)$. In order to determine $\partial_{j} f(a), f$ needs to be defined on the points $a+h \mathbf{e}_{j}, a$ and $a-h \mathbf{e}_{j}$. Surprisingly, since $\partial_{j}^{2}=\Delta_{j}^{+} \Delta_{j}^{-}$, the action of $\partial_{j}^{2}$ on $f$ in $a$ only requires the same three points, while for $\partial_{j}^{3}$, we will need the values of $f$ in two extra points: $a+2 h \mathbf{e}_{j}$ and $a-2 h \mathbf{e}_{j}$.

Thus, if we are interested in calculating $\partial_{\ell_{1}} \ldots \partial_{\ell_{k}} f(a)$, we rewrite $\left\{\ell_{1}, \ldots, \ell_{k}\right\}$ as $\underline{\alpha}=\left(\alpha_{1}, \ldots, \alpha_{m}\right)$ where the number of times that $j$ appears in $\left\{\ell_{1}, \ldots, \ell_{k}\right\}$ is equal to $\alpha_{j}(j=1, \ldots, m)$, i.e. we regroup the factors by their index. Then in order for $\partial_{\ell_{1}} \ldots \partial_{\ell_{k}} f(a)= \pm \partial_{1}^{\alpha_{1}} \ldots \partial_{m}^{\alpha_{m}} f(a)$ to be defined, $f$ needs to be defined on the points:

$$
\left\{a+x:\left|x_{j}\right| \leqslant\left\lceil\frac{\alpha_{j}}{2}\right\rceil h, j=1, \ldots, m\right\}=\left\{x:\left|x_{j}-a_{j}\right| \leqslant\left\lceil\frac{\alpha_{j}}{2}\right\rceil h, j=1, \ldots, m\right\}
$$

where $\lceil x\rceil$ is the smallest integer not less than $x$. Conversely, if $f$ is defined on the $m$-dimensional rectangle $\left\{x:\left|x_{j}-a_{j}\right| \leqslant \ell_{j} h\right\}$ centered at $a$, where $\ell_{1}, \ldots, \ell_{m}$ are given natural numbers, then all 
finite differences $\partial_{1}^{\alpha_{1}} \ldots \partial_{m}^{\alpha_{m}} f(a)$ with $\alpha_{j} \leqslant 2 \ell_{j}, j=1, \ldots, m$ can be calculated. Since for a point $x$ in the given set $\Omega$, all terms $\partial_{1}^{\alpha_{1}} \ldots \partial_{m}^{\alpha_{m}} f(0)$ with $\alpha_{j}<2 \frac{\left|x_{j}\right|}{h}+1, j=1, \ldots, m$ need to be explicitly calculated, $f$ needs to be defined on

$$
\left\{y:\left|y_{j}\right| \leqslant\left\lceil\frac{\alpha_{j}}{2}\right\rceil h\right\}=\left\{y:\left|y_{j}\right|<\left\lceil\frac{\left|x_{j}\right|}{h}+\frac{1}{2}\right\rceil h\right\}=\left\{y:\left|y_{j}\right| \leqslant\left|x_{j}\right|\right\}
$$

In conclusion, for each point $x \in \Omega$ we have to consider also the reflection of $x$ with respect to all axes, and the function $f$ has to be defined on the union of all resulting $m$-dimensional rectangular prisms.

Example 3.1. Let $\Omega$ be the depicted set at the left. In order to be able to write down, even formally, the series (7) in the point $(2,3), f$ has to be defined on all points of the rectangle centered at the origin which is depicted at the right.
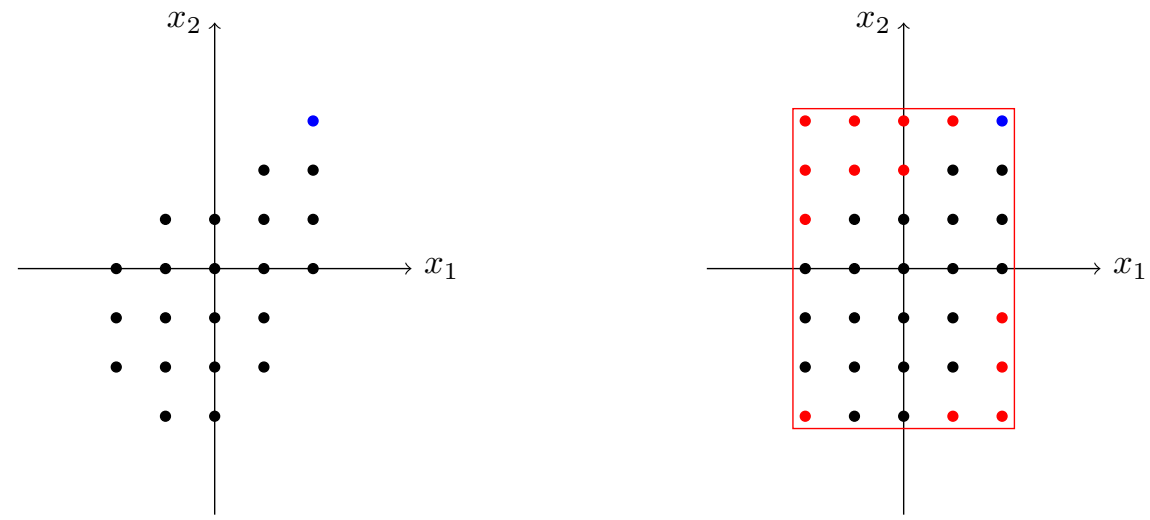

Example 3.2. Let $\Omega$ be the depicted set at the left. Writing down the series (7) in each point of $\Omega$ requires that $f$ is defined on the union of the two rectangles at the right.
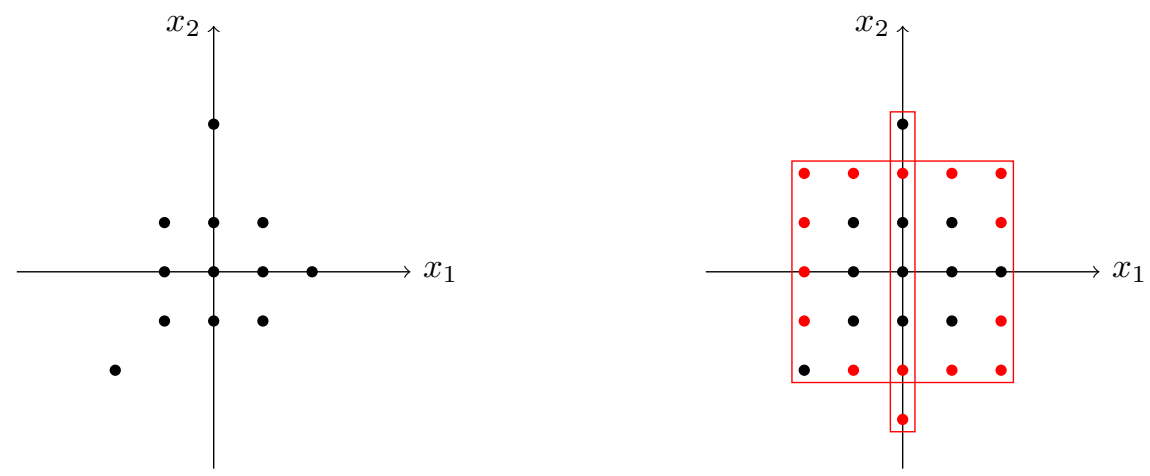

On the other hand, when the function $f$ is defined on the bounded set $\Omega$ only, then we will only be able to write down the series (7) for those points $x \in \Omega$ for which the corresponding $m$-dimension rectangular prism $\left\{y:\left|y_{j}\right| \leqslant\left|x_{j}\right|\right\}$ is contained in $\Omega$.

Example 3.3. In the following examples, the series (7) is defined only in those points $x$ which are circled. 

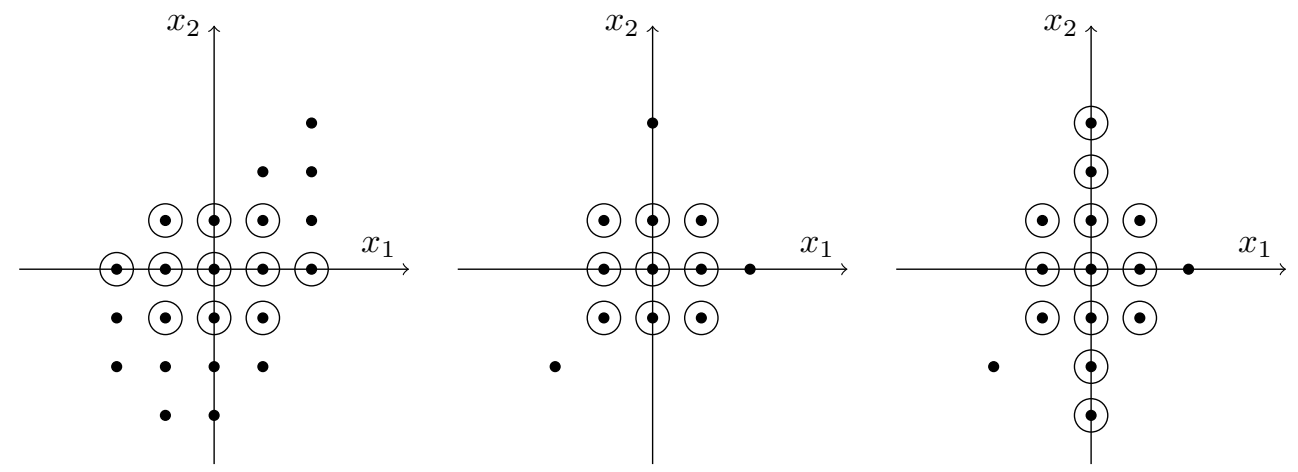

\subsection{Convergence of the series}

We now adress the fundamental question of convergence of the series (7).

Consider a point $x$ in $\Omega$; on account of (8), the only non-vanishing terms $\xi_{1}^{\alpha_{1}} \ldots \xi_{m}^{\alpha_{m}}[1](x)$ are those for which $\alpha_{j} \leqslant 2 \frac{\left|x_{j}\right|}{h}$ for all $j=1, \ldots, m$. For any point $x \in \Omega$ the series thus reduces to a finite sum, whence its pointwise convergence is ensured for any $x$ for which it is defined. However, the Taylor series expansion of a monogenic function in the continuous setting not only converges pointwise, but also shows so-called 'normal convergence'. For the discrete Taylor series expansion, we can prove a similar convergence theorem, which we will also refer to as 'normal convergence'. For this however, we first need an auxiliary result.

Lemma 3.1. Consider a bounded subset $K \subset \mathbb{Z}_{h}^{m}$, then there exists an $N_{K} \in \mathbb{N}$ s.t. for every multi-index $\underline{\alpha} \in \mathbb{N}^{m}$ with $|\underline{\alpha}| \geqslant N_{K}$

$$
\xi_{1}^{\alpha_{1}} \ldots \xi_{m}^{\alpha_{m}}[x]=0, \forall x \in K
$$

Proof. This directly follows from (8).

Lemma 3.2 (Normal convergence). Consider a bounded subset $K \subseteq \Omega$. Then, the terms of the multiple power series being ordered in an arbitrary way for a single summation index $j$, it holds that

$$
\sum_{j=J_{1}}^{J_{2}} \sup _{x \in K}\left(\frac{1}{k !} \xi_{\ell_{1}} \ldots \xi_{\ell_{k}}[x] \partial_{\ell_{k}} \ldots \partial_{\ell_{1}} f(0)\right) \longrightarrow 0
$$

if $\inf \left(J_{1}, J_{2}\right) \rightarrow+\infty$.

Proof. Lemma 3.1 states that there exists a natural number $N_{K}$ s.t. $\xi_{1}^{\alpha_{1}} \ldots \xi_{m}^{\alpha_{m}}[x]=0$, for all $x \in$ $K$ and each multi-index $\underline{\alpha} \in \mathbb{N}^{m}$ with $|\underline{\alpha}| \geqslant N_{K}$. Thus, for each $k$-tuple $\left(\ell_{1}, \ldots, \ell_{k}\right) \in\{1, \ldots, m\}^{k}$ with $k \geqslant N_{K}$, we have

$$
\sup _{x \in K}\left(\frac{1}{k !} \xi_{\ell_{1}} \ldots \xi_{\ell_{k}}[x] \partial_{\ell_{k}} \ldots \partial_{\ell_{1}} f(0)\right)=0, \quad \forall x \in K
$$

Now, we choose an ordering of the terms of the multiple power series. Considering the finite number (say $r$ ) of elements $\xi_{\ell_{1}} \ldots \xi_{\ell_{k}}$ with $k \leqslant N_{K}$, let us denote the respective places on which these elements appear in the ordering by $s_{1}, \ldots, s_{r}$. Then it holds that

$$
\sum_{j=J_{1}}^{J_{2}} \sup _{x \in K}\left(\frac{1}{k !} \xi_{\ell_{1}} \ldots \xi_{\ell_{k}}[x] \partial_{\ell_{k}} \ldots \partial_{\ell_{1}} f(0)\right)=0 \text { if } \inf \left(J_{1}, J_{2}\right)>\max \left\{s_{1}, \ldots, s_{r}\right\}
$$




\subsection{Convergence of the derived series}

When considering a given discrete function $f$, we also need to be able to determine the action of one or more co-ordinate finite difference operators $\partial_{j}$ on it. When this discrete function is given in the form of a Taylor series expansion, we thus need to be able to consider the 'derived' series. However, this has some consequences on the conditions imposed on the domain $\Omega$, as we will see below.

\subsubsection{Domain of the derived series}

The action of the finite difference operator $\partial_{j}$ on (7) in an arbitrary point $x \in \Omega$ yields

$$
\sum_{k=1}^{\infty} \sum_{|\underline{\alpha}|=k} \frac{(-1)^{\alpha_{1}+\ldots+\alpha_{j-1}}}{\alpha_{1} ! \ldots \alpha_{m} !} \xi_{1}^{\alpha_{1}} \ldots \xi_{j-1}^{\alpha_{j-1}} \xi_{j}^{\alpha_{j}-1} \xi_{j+1}^{\alpha_{j+1}} \ldots \xi_{m}^{\alpha_{m}}[1](x) \partial_{m}^{\alpha_{m}} \ldots \partial_{j}^{\alpha_{j}} \ldots \partial_{1}^{\alpha_{1}} f(0)
$$

where only the terms $\xi_{1}^{\alpha_{1}} \ldots \xi_{j}^{\alpha_{j}-1} \ldots \xi_{m}^{\alpha_{m}}[1](x)$ with $\alpha_{i} \leqslant 2 \frac{\left|x_{i}\right|}{h}(i \neq j)$ and $\alpha_{j} \leqslant 2 \frac{\left|x_{j}\right|}{h}+1$ will differ from zero. In (7) these terms are combined with the finite differences $\partial_{m}^{\alpha_{m}} \ldots \partial_{j}^{\alpha_{j}} \ldots \partial_{1}^{\alpha_{1}} f(0)$, whence it is necessary that all $\partial_{m}^{\alpha_{m}} \ldots \partial_{j}^{\alpha_{j}} \ldots \partial_{1}^{\alpha_{1}} f(0)$ with $\alpha_{i} \leqslant 2 \frac{\left|x_{i}\right|}{h}(i \neq j)$ and $\alpha_{j} \leqslant 2 \frac{\left|x_{j}\right|}{h}+1$ are defined. In order to evaluate the derived series (9) in the point $x \in \Omega$, the function thus needs to be defined on

$$
\left\{y:\left|y_{j}\right| \leqslant\left\lceil\frac{\alpha_{j}}{2}\right\rceil h \leqslant\left|x_{j}\right|+h,\left|y_{i}\right| \leqslant\left\lceil\frac{\alpha_{i}}{2}\right\rceil h \leqslant\left|x_{i}\right|(i \neq j)\right\}
$$

If we want to consider the derived series (9) on the whole of $\Omega$, the function $f$ thus needs to be defined on the following set of points around zero:

$$
\bigcup_{x \in \Omega}\left\{y:\left|y_{j}\right| \leqslant\left|x_{j}\right|+h,\left|y_{i}\right| \leqslant\left|x_{i}\right|(i \neq j)\right\}
$$

With the application of each additional finite difference operator $\partial_{j}, f$ must be defined on an extra set of points in the $j$ direction.
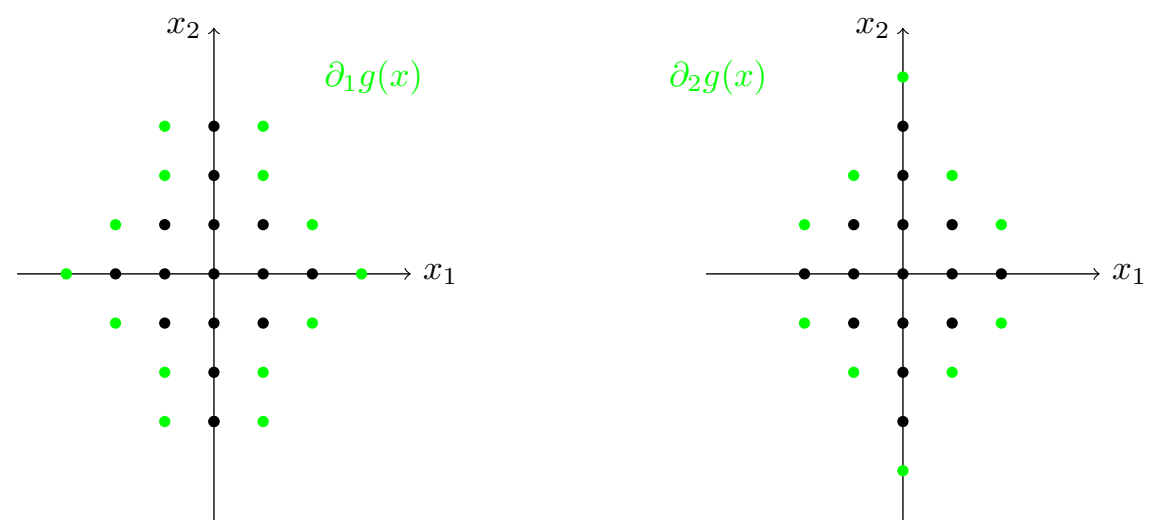

Since, again, for every point there only remain a finite number of terms, any derived series will thus converge both pointwise and normally, wherever it is defined. 


\subsection{Equivalence of discrete functions}

At this moment, we only can say that, if the discrete function $f$ can be written in the form of a Taylor series expansion, it has to be the series (7). However, we still explicitly need to check that $f$ and (7) are equivalent in $\Omega$.

We will show this by considering "a sufficient number" of finite differences of both functions in an arbitrary point $x$ in $\Omega$ and showing that those finite differences are equal, from which the equivalence of the two functions will follow. To this end we formulated some technical auxiliary results, lemma A.1-A.3, given in the appendix. The main result of this section then reads as follows.

Theorem 3.1. Let $x=\left(x_{1}, \ldots, x_{m}\right)$ be an arbitrary, but fixed point. If it holds that

$$
\partial_{1}^{\alpha_{1}} \ldots \partial_{m}^{\alpha_{m}} f(0)=0, \quad \text { for all } \alpha_{i} \leqslant 2 \frac{\left|x_{i}\right|}{h}, i=1, \ldots, m
$$

then the discrete function $f$ is zero on the whole rectangle $\left\{y:\left|y_{i}\right| \leqslant\left|x_{i}\right|, i=1, \ldots, m\right\}$.

Proof. Repeated combination of Lemma A.1 and Lemma A.2 reveals that the function values in all points of the rectangle are zero.

Corollary 3.1. Let $a=\left(a_{1}, \ldots, a_{m}\right)$ and $x=\left(x_{1}, \ldots, x_{m}\right)$ be arbitrary, but fixed points,. If it holds that

$$
\partial_{1}^{\alpha_{1}} \ldots \partial_{m}^{\alpha_{m}} f(a)=0, \quad \text { for all } \alpha_{i} \leqslant 2 \frac{\left|x_{i}-a_{i}\right|}{h}, i=1, \ldots, m
$$

then $f$ is zero on the whole rectangle $\left\{y:\left|y_{i}-a_{i}\right| \leqslant\left|x_{i}-a_{i}\right|, i=1, \ldots, m\right\}$.

Having now established conditions under which we may conclude the equivalence of two discrete functions, we will apply these conditions to a discrete function $f$ and its Taylor series expansion $g$, (7). The function $f$ is, as before, defined on the bounded set $\Omega$, containing the origin, submitted to the geometric condition that

$$
\left\{y \in \mathbb{Z}_{h}^{m}:\left|y_{j}\right| \leqslant\left|x_{j}\right|\right\} \subseteq \Omega, \quad \forall x \in \Omega
$$

On account of the above results, the function $f$ and the corresponding series $g$ will be equivalent on $\Omega$, if it holds, for all $x$ in $\Omega$, that

$$
\partial_{1}^{\alpha_{1}} \ldots \partial_{m}^{\alpha_{m}}(f-g)(0)=0, \quad \text { for all } \alpha_{i} \leqslant 2 \frac{\left|x_{i}\right|}{h}, i=1, \ldots, m
$$

Rewriting the Taylor series expansion as

$$
\sum_{k=0}^{\infty} \sum_{|\underline{r}|=k} \frac{1}{r_{1} ! \ldots r_{m} !} \xi_{1}^{r_{1}} \ldots \xi_{m}^{r_{m}}[1](x) \partial_{m}^{r_{m}} \ldots \partial_{1}^{r_{1}} f(0)
$$

it may be checked by direct calculation that this condition indeed is fulfilled, whence any discrete function $f$ will be equivalent to its Taylor series expansion, in all points were this Taylor series expansion is defined.

To conclude this section, it is worth observing a few differences with the continuous setting:

(i) In the discrete setting, the order of the co-ordinate difference operators appearing in the Taylor series expansion is not random. Switching two co-ordinate difference operators in a term of the series influences the sign of that term, since these co-ordinate difference operators anti-commute. 
(ii) In [1, Chapter 2.11.3], one obtains the Taylor series expansion of a real-analytic function by splitting this function into its real-valued components and expanding each component into its separate Taylor series expansion, which are then recombined. In the discrete setting, it makes no sense to first split the function $f$ into its components, since these are then combined with $\xi_{\ell_{1}} \ldots \xi_{\ell_{k}}$, which are not scalar.

(iii) There are no conditions on the function $f$ in order for the Taylor series expansion to be defined, unlike the continuous setting, where the function needs to be real-analytic.

\subsection{The Taylor series expansion of a discrete monogenic function}

We already mentioned, see Definition 2.2, that the so-called Fueter polynomials $V_{\ell_{1}, \ldots, \ell_{k}}, \ell_{j} \in$ $\{2, \ldots, m\}$, constitute a basis for the space $\mathcal{M}_{k}^{(m)}$ of discrete spherical monogenics of degree $k$.

In the continuous setting it has been possible, see [1, Chapter 2.11 (Theorem 11.3.4)], to rewrite the Taylor series expansion of a monogenic function in terms of the Fueter polynomials, which constitute a basis for the space of spherical monogenics. It thus is a logical question to examine whether we may also rewrite the discrete Taylor series expansion of a discrete monogenic function in such a way that the discrete Fueter polynomials appear.

For any discrete function, grouping the terms of its Taylor series expansion, we already may rewrite it in the following form:

$$
f(x)=\sum_{k=0}^{\infty} P_{k} f(x)
$$

where $P_{k} f(x)$ stands for the discrete homogeneous polynomial of degree $k$, given by

$$
P_{k} f(x)=\frac{1}{k !} \sum_{\ell_{1}=0}^{m} \ldots \sum_{\ell_{k}=0}^{m} \xi_{\ell_{1}} \ldots \xi_{\ell_{k}}[1](x) \partial_{\ell_{k}} \ldots \partial_{\ell_{1}} f(0)
$$

Considering (10) as a series of polynomials it is clear that the corresponding series of numbers

$$
\sum_{k=0}^{\infty} \sup _{x \in K} P_{k} f(x)
$$

is convergent, for each bounded set $K \subset \Omega$. Similar properties clearly hold for any of its 'derived' series as well, provided that these are defined.

From now on we assume that $f$ is left discrete monogenic. We first prove an auxiliary result.

Lemma 3.3. Let $\Omega$ be a bounded set containing the origin such that, for all $x \in \Omega$ the corresponding rectangle $\left\{y:\left|y_{j}\right| \leqslant\left|x_{j}\right|\right\} \subseteq \Omega$, and let $f$ be defined on the set

$$
\bigcup_{x \in \Omega}\left\{y:\left|y_{j}\right| \leqslant\left|x_{j}\right|+h\right\}
$$

If $f$ is left discrete monogenic in $\Omega$, then for each $k \in \mathbb{N}$ the polynomial $P_{k} f,(11)$, is a discrete spherical monogenic of degree $k$.

Proof. By the condition imposed on $\Omega$, the Taylor series expansion of the discrete function $f$ is defined and equivalent to $f$ in $\Omega$, i.e. (10) holds for all $x \in \Omega$. Furthermore, the assumption that $f$ is defined on the set (12), ensures that we can consider the action of the Dirac operator $D$ on 
$P_{k} f(x)$ for all $x \in \Omega$. The resulting polynomial $D\left(P_{k} f\right)$ is discrete homogeneous of degree $k-1$, whence we can write it in terms of the standard discrete homogeneous polynomials

$$
D\left(P_{k} f\right)=\sum_{\alpha_{1}+\ldots+\alpha_{m}=k-1} \xi_{1}^{\alpha_{1}} \ldots \xi_{m}^{\alpha_{m}}[1](x) \lambda_{\alpha_{1}, \ldots, \alpha_{m}}
$$

with, for the moment, unknown coefficients $\lambda_{\alpha_{1}, \ldots, \alpha_{m}}$. In order to conclude that $P_{k} f$ is monogenic, it thus suffices to prove that all $\lambda_{\alpha_{1}, \ldots, \alpha_{m}}$ equal zero. To this end, take $\beta=\left(\beta_{1}, \ldots, \beta_{m}\right)$ with $|\beta|=k-1$, and consider the differential operator $\partial_{m}^{\beta_{m}} \ldots \partial_{1}^{\beta_{1}}$. Since already $D f=0$ in $\Omega$, also $\partial_{m}^{\beta_{m}} \ldots \partial_{1}^{\beta_{1}} D f=0$ in $\Omega$, which, in view of the decomposition (10), yields

$$
\begin{aligned}
0 & =\partial_{m}^{\beta_{m}} \ldots \partial_{1}^{\beta_{1}} D\left(P_{k} f\right)+\text { discrete homogeneous terms of degree } \geq 1 \\
& =\beta_{1} ! \ldots \beta_{m} ! \lambda_{\beta_{1}, \ldots, \beta_{m}}+\text { discrete homogeneous terms of degree } \geq 1
\end{aligned}
$$

When evaluated at the origin, this equality reduces to $0=\beta_{1} ! \ldots \beta_{m} ! \lambda_{\beta_{1}, \ldots, \beta_{m}}$. As $\underline{\beta}$ was arbitrarily chosen, all coefficients $\lambda_{\beta_{1}, \ldots, \beta_{m}}$ with $|\beta|=k-1$ indeed are zero, whence $P_{k} f$ is discrete monogenic.

The main result of this section then reads as follows.

Theorem 3.2. Let $\Omega$ be a bounded set as in Lemma 3.3 and let the function $f$ be defined on the set (12). If $f$ is left discrete monogenic in $\Omega$, then $f$ can be developed into a convergent series of spherical monogenics as follows:

$$
f(x)=\sum_{k=0}^{\infty}\left(\sum_{\left(\ell_{1}, \ldots, \ell_{k}\right)} V_{\ell_{1}, \ldots, \ell_{k}} \partial_{\ell_{k}} \ldots \partial_{\ell_{1}} f(0)\right)
$$

Moreover, any derived series (whenever it is defined) is normally convergent in $\Omega$ to the corresponding 'derivative' of $f$, i.e., if $j$ denotes a single summation index, the terms of the multiple power series being ordered in an arbitrary way, then for each bounded $K \subset \Omega$ and each multi-index $\underline{\beta}=\left(\beta_{1}, \ldots, \beta_{m}\right) \in \mathbb{N}^{m}$, it holds that

$$
\sum_{j=J}^{J^{\prime}} \sup _{x \in K}\left(\partial_{1}^{\beta_{1}} \ldots \partial_{m}^{\beta_{m}}\left(V_{\ell_{1}, \ldots, \ell_{k}} \partial_{\ell_{k}} \ldots \partial_{\ell_{1}} f(0)\right)\right) \rightarrow 0 \text { if } \inf \left(J, J^{\prime}\right) \rightarrow \infty
$$

Proof. The imposed conditions on $\Omega$ ensure that the Taylor series expansion of $f$ is defined, and can be written in the form (10), where, on account of the assumed discrete monogenicity of $f$ and Lemma 3.3, the polynomials $P_{k} f(x)$ are (left) discrete spherical monogenics of degree $k$. Now, in general, a discrete spherical monogenic $Q_{k}$ of degree $k$ can be expanded in terms of the Fueter polynomials of degree $k$ [7, Lemma 5], more precisely:

$$
Q_{k}(x)=\sum_{\left(\ell_{1}, \ldots, \ell_{k}\right)} V_{\ell_{1}, \ldots, \ell_{k}}(x) \partial_{\ell_{k}} \ldots \partial_{\ell_{1}} Q_{k}(x)
$$

where repetitions of the $\ell_{j}$ are allowed, but where every $k$-tuple $\left(\ell_{1}, \ldots, \ell_{k}\right)$ only appears once in the sum. In the case of the polynomials $P_{k} f(x)$ this becomes

$$
P_{k} f(x)=\sum_{\left(\ell_{1}, \ldots, \ell_{k}\right)} V_{\ell_{1}, \ldots, \ell_{k}}(x) \partial_{\ell_{k}} \ldots \partial_{\ell_{1}}\left(P_{k} f\right)(x)
$$

with $\partial_{\ell_{k}} \ldots \partial_{\ell_{1}}\left(P_{k} f\right)(x)=\partial_{\ell_{k}} \ldots \partial_{\ell_{1}} f(0)$, from which the expansion (13) follows. 
Remark 3.1. We may also consider the series

$$
\sum_{k=0}^{\infty} \sum_{\left(\ell_{1}, \ldots, \ell_{k}\right)} V_{\ell_{1}, \ldots, \ell_{k}} \partial_{\ell_{k}} \ldots \partial_{\ell_{1}} f(0)
$$

meaning that we do not group the terms according to $k$. Now, if $N_{\Omega}$ is the natural number from Lemma 3.1, such that for all $x$ in $\Omega$ and all multi-indices $|\underline{\alpha}| \geqslant N_{\Omega}$ we have $\xi_{1}^{\alpha_{1}} \ldots \xi_{m}^{\alpha_{m}}[x]=0$, then we also have that $V_{\ell_{1}, \ldots, \ell_{k}}(x)=0$ for all $x \in \Omega$ and for all $k \geqslant N_{\Omega}$. So both in the series (13) and in the above series, there are only a finite number of non-vanishing terms, implying that both will converge pointwise as well as normally on $\Omega$.

\subsection{Series of discrete spherical monogenics}

The previous results have revealed that every discrete monogenic function may be expressed in terms of the discrete Fueter polynomials.

On the other hand, series of discrete spherical monogenics may in turn be related to discrete monogenic functions, as stated in the following theorem.

Theorem 3.3 (Converse of Theorem 3.2). Let $\Omega$ be a bounded set as in Lemma 3.3. If the series of discrete spherical monogenics

$$
\sum_{k=0}^{\infty} P_{k}(x)=\sum_{k=0}^{\infty}\left(\sum_{\left(\ell_{1}, \ldots, \ell_{k}\right)} V_{\ell_{1}, \ldots, \ell_{k}} \lambda_{\ell_{1}, \ldots, \ell_{k}}\right)
$$

is defined on the set (12), then it represents in $\Omega$ a (left) discrete monogenic function $f$. Moreover, it then precisely is the Taylor series expansion about the origin of this function $f$ in $\Omega$.

Proof. First of all, the series (14) converges to a function $f$ on the bounded set $\Omega$, in view of Lemma 3.1. Since moreover this function $f$ thus is defined on the set (12), we can consider the action of the Dirac operator on it in all points of $\Omega$. Clearly this action yields zero, since the series reduces to finite sum of discrete monogenic terms. Hence the function $f$ indeed is discrete monogenic, whence its Taylor series expansion may be written as:

$$
f(x)=\sum_{k=0}^{\infty} \sum_{\left(\ell_{1}, \ldots, \ell_{k}\right)} V_{\ell_{1}, \ldots, \ell_{k}}(x) \partial_{\ell_{k}} \ldots \partial_{\ell_{1}} f(0), \forall x \in \Omega
$$

The action of the finite difference operator $\partial_{\ell_{k}} \ldots \partial_{\ell_{1}}$ on both sides of the resulting equality

$$
\sum_{\left(\ell_{1}, \ldots, \ell_{k}\right)} V_{\ell_{1}, \ldots, \ell_{k}} \partial_{\ell_{k}} \ldots \partial_{\ell_{1}} f(0)=\sum_{\left(\ell_{1}, \ldots, \ell_{k}\right)} V_{\ell_{1}, \ldots, \ell_{k}} \lambda_{\ell_{1}, \ldots, \ell_{k}}
$$

directly reveals that $\lambda_{\ell_{1}, \ldots, \ell_{k}}=\partial_{\ell_{k}} \ldots \partial_{\ell_{1}} f(0)$, so that (14) indeed coincides with the Taylor series expansion of $f$.

\section{Taylor series expansion about a general point}

Given a discrete function $f$ defined on a set $\Omega$, we clearly are not limited to expanding $f$ into a discrete Taylor series expansion about the origin exclusively; any point contained in $\Omega$ may qualify, under some geometric conditions. 
So, let the point $a \in \Omega$ be such that for all $x \in \Omega$ the corresponding set

$$
\left\{y:\left|y_{j}-a_{j}\right| \leqslant\left|x_{j}-a_{j}\right|, j=1, \ldots, m\right\}
$$

entirely belongs to $\Omega$. Then consider the translated set $\Omega^{\prime}=\{x-a \mid x \in \Omega\}=\{y \mid y+a \in \Omega\}$ and associate to any discrete function $f$ defined on $\Omega$ the discrete function $f^{\prime}$ on $\Omega^{\prime}$ by $f^{\prime}(y)=f(y+a)$ for all $y \in \Omega^{\prime}$, or equivalently, $f(x)=f^{\prime}(x-a)$ for all $x \in \Omega$. The Taylor series expansion of the function $f^{\prime}$ in $\Omega^{\prime}$ is well-defined, namely

$$
f^{\prime}(y)=\sum_{k=0}^{\infty} \frac{1}{k !} \sum_{\ell_{1}=1}^{m} \ldots \sum_{\ell_{k}=1}^{m} \xi_{\ell_{1}} \ldots \xi_{\ell_{k}}[1](y) \partial_{\ell_{k}} \ldots \partial_{\ell_{1}} f^{\prime}(0)
$$

Observe that $\partial_{\ell_{k}} \ldots \partial_{\ell_{1}} f^{\prime}(0)=\partial_{\ell_{k}} \ldots \partial_{\ell_{1}} f(a)$, whence

$$
f(x)=\sum_{k=0}^{\infty} \frac{1}{k !} \sum_{\ell_{1}=1}^{m} \ldots \sum_{\ell_{k}=1}^{m} \xi_{\ell_{1}} \ldots \xi_{\ell_{k}}[1](x-a) \partial_{\ell_{k}} \ldots \partial_{\ell_{1}} f(a)
$$

for $f$ defined on $\Omega$. If moreover $f$ is (left) discrete monogenic on $\Omega$, then its Taylor series expansion about a general point $a$ can also be rewritten by means of the Fueter polynomials:

$$
f(x)=\sum_{k=0}^{\infty} \sum_{\left(\ell_{1}, \ldots, \ell_{k}\right)} V_{\ell_{1}, \ldots, \ell_{k}}(x-a) \partial_{\ell_{k}} \ldots \partial_{\ell_{1}} f(a)
$$

\section{Taylor series expansion of the discrete delta function in one dimension}

As an illustrative example, we apply the theory of discrete Taylor series expansion to the discrete delta function in one dimension on the standard grid $(h=1)$ :

$$
\delta_{j}(x)=\left\{\begin{array}{lc}
1, & x=j \mathbf{e} \\
0, & \text { otherwise }
\end{array}, \quad j \in \mathbb{Z}\right.
$$

where we have denoted $\mathbf{e}_{1} \equiv \mathbf{e}$, and we will, likewise, also drop the subscript 1 in all other notations. For all $j \in \mathbb{Z}, \delta_{j}$ is defined on the whole of $\mathbb{Z}$; we may thus also consider the Taylor series expansion of $\delta_{j}$ on the whole of $\mathbb{Z}$. It is given by

$$
\delta_{j}(x)=\sum_{\ell=0}^{\infty} \frac{1}{\ell !} \xi^{\ell}[1]\left[\partial^{\ell} \delta_{j}(u)\right]_{u=0}
$$

In order to determine the values $\left[\partial^{\ell} \delta_{j}(u)\right]_{u=0}$ we use the following result, which is obtained by direct calculation.

Lemma 5.1. For all $k \in \mathbb{N}$, we have that

$$
\begin{aligned}
\partial^{2 k} \delta_{j} & =\sum_{i=0}^{2 k}(-1)^{i}\left(\begin{array}{c}
2 k \\
i
\end{array}\right) \delta_{j-(k-i)} \\
\partial^{2 k+1} \delta_{j} & =\mathbf{e}^{+} \sum_{i=0}^{2 k+1}(-1)^{i}\left(\begin{array}{c}
2 k+1 \\
i
\end{array}\right) \delta_{j-(k+1-i)}+\mathbf{e}^{-} \sum_{i=0}^{2 k+1}(-1)^{i}\left(\begin{array}{c}
2 k+1 \\
i
\end{array}\right) \delta_{j-(k-i)}
\end{aligned}
$$


We will now examine the results (16)-(17) carefully, in view of their evaluation in the origin.

First consider (17). We will have to distinguish between $j>0, j=0$ and $j<0$. The first term of $\partial^{2 k+1} \delta_{j}$ includes $\delta_{j-k-1}, \ldots, \delta_{j+k}$, while the second term includes $\delta_{j-k}, \ldots, \delta_{j+k+1}$. Hence, for positive $j, \partial^{2 k+1} \delta_{j}$ contains $\delta_{0}$ in the first term when $j \leqslant k+1$ and in the second term when $j \leqslant k$. Clearly, all $\partial^{2 k+1} \delta_{0}$ contain $\delta_{0}$. For negative $j$, the first term contains $\delta_{0}$ if $k \geqslant|j|$ and the second term contains $\delta_{0}$ if $k+1 \geqslant|j|$. Next, consider (16). Here we note that $\partial^{2 k} \delta_{j}$ includes $\delta_{j-k}, \ldots, \delta_{j+k}$, hence it contains $\delta_{0}$ when $|j| \leqslant k$. In conclusion, we have the following:

$$
\begin{gathered}
{\left[\partial^{2 k} \delta_{j}(u)\right]_{u=0}=(-1)^{k-j}\left(\begin{array}{c}
2 k \\
k-j
\end{array}\right)} \\
{\left[\partial^{2 k+1} \delta_{j}(u)\right]_{u=0}= \begin{cases}\mathbf{e}^{+}, & j=k+1 \\
(-1)^{k+1-j}\left(\begin{array}{c}
2 k+1 \\
k+1-j
\end{array}\right)\left(\mathbf{e}^{+}-\mathbf{e}^{-}\right), & 0 \leqslant j \leqslant k \\
-\mathbf{e}^{-}, & j=-(k+1)\end{cases} }
\end{gathered}
$$

Substitution of these values in (15) yields the explicit form of the discrete Taylor series expansion of the delta function. This is the subject of the following theorem.

Theorem 5.1 (Taylor series expansion of $\delta_{j}$ ). For $j>0$, the Taylor series expansion of the corresponding delta function is given by

$$
\begin{aligned}
\delta_{j}(x)= & \sum_{\ell=j}^{|x|} \frac{(-1)^{\ell-j}}{(\ell-j) !(\ell+j) !} \xi^{2 \ell}[1](x)+\frac{\xi^{2 j-1}[1](x)}{(2 j-1) !} \mathbf{e}^{+} \\
& +\sum_{\ell=j}^{|x|-1} \frac{(-1)^{\ell+1-j}}{(\ell-j) !(\ell+j) !} \xi^{2 \ell+1}[1](x)\left(\frac{\mathbf{e}^{+}}{(\ell-j+1)}-\frac{\mathbf{e}^{-}}{(\ell+j+1)}\right)
\end{aligned}
$$

while for $j<0$ it is given by

$$
\begin{aligned}
\delta_{j}(x)= & \sum_{\ell=|j|}^{|x|} \frac{(-1)^{\ell-j}}{(\ell-j) !(\ell+j) !} \xi^{2 \ell}[1](x)-\frac{\xi^{2|j|-1}[1](x)}{(2|j|-1) !} \mathbf{e}^{-} \\
& +\sum_{\ell=|j|}^{|x|-1} \frac{(-1)^{\ell+1-j}}{(\ell-j) !(\ell+j) !} \xi^{2 \ell+1}[1](x)\left(\frac{\mathbf{e}^{+}}{(\ell-j+1)}-\frac{\mathbf{e}^{-}}{(\ell+j+1)}\right)
\end{aligned}
$$

Finally, for $j=0$ we have

$$
\delta_{0}(x)=\sum_{\ell=0}^{|x|} \frac{(-1)^{\ell}}{(\ell !)^{2}} \xi^{2 \ell}[1](x)+\sum_{\ell=0}^{|x|-1} \frac{(-1)^{\ell+1}}{\ell !(\ell+1) !} \xi^{2 \ell+1}[1](x)\left(\mathbf{e}^{+}-\mathbf{e}^{-}\right)
$$

Example 5.1. By direct calculation one can easily check that

$$
\delta_{0}(0)=\sum_{\ell=0}^{\infty} \frac{(-1)^{\ell}}{(\ell !)^{2}} \xi^{2 \ell}[1](0)+\sum_{\ell=0}^{\infty} \frac{(-1)^{\ell+1}}{\ell !(\ell+1) !} \xi^{2 \ell+1}[1](0)\left(\mathbf{e}^{+}-\mathbf{e}^{-}\right)=1 .
$$


while

$$
\begin{aligned}
\delta_{0}(1) & =\sum_{\ell=0}^{1} \frac{(-1)^{\ell}}{(\ell !)^{2}} \xi^{2 \ell}[1](1)+\sum_{\ell=0}^{0} \frac{(-1)^{\ell+1}}{\ell !(\ell+1) !} \xi^{2 \ell+1}[1](1)\left(\mathbf{e}^{+}-\mathbf{e}^{-}\right) \\
& =1-\xi^{2}[1](1)-\xi[1](1)\left(\mathbf{e}^{+}-\mathbf{e}^{-}\right)=0 \\
\delta_{0}(-1) & =\sum_{\ell=0}^{1} \frac{(-1)^{\ell}}{(\ell !)^{2}} \xi^{2 \ell}[1](-1)+\sum_{\ell=0}^{0} \frac{(-1)^{\ell+1}}{\ell !(\ell+1) !} \xi^{2 \ell+1}[1](-1)\left(\mathbf{e}^{+}-\mathbf{e}^{-}\right) \\
& =1-\xi^{2}[1](-1)-\xi[1](-1)\left(\mathbf{e}^{+}-\mathbf{e}^{-}\right)=0
\end{aligned}
$$

\section{Application: the sampling theorem}

In 1948 and 1949, Claude E. Shannon published two papers [11, 12] in which he stated the socalled 'sampling theorem' for lowpass functions, which plays an important role in the information theory, as a connecting link between continuous-time and discrete-time signals. In the first paper of Shannon [11], the theorem ('Theorem 13') is formulated as follows.

Theorem 6.1. Let $f(t)$ contain no frequencies higher than $W$. Then

$$
f(t)=\sum_{n=-\infty}^{\infty} X_{n} \frac{\sin (\pi(2 W t-n))}{\pi(2 W t-n)}, \text { with } X_{n}=f\left(\frac{n}{2 W}\right)
$$

The theorem thus states that if a function contains no frequencies higher than a given $W$ cps (cycles per second), then it is completely determined by giving its ordinates at a series of sampling points spaced $\frac{1}{2 W}$ seconds apart, a fact which is common knowledge in the communication art. Intuitively, one can say that if a function $f(t)$ contains no frequencies higher than $W$, it cannot change to a substantially new value in a time less than one-half cycle of the highest frequency, i.e. $\frac{1}{2} W$.

In this section we will demonstrate the link between this decomposition and the decomposition of a discrete function $f$ by means of discrete delta functions.

\subsection{Continuous expansion of a discrete delta function}

A discrete function $f(x)$ in one dimension clearly is completely determined by its values in the integer points $n$, whence it can be written as

$$
f(x)=\sum_{n=-\infty}^{\infty} f(n) \delta_{n}(x)
$$

We will now establish a link between the discrete delta functions $\delta_{n}$ and the continuous sinc functions, evaluated in equidistant sampling points (the integers). 
Starting from the Taylor series expansion of $\delta_{0}$, we define a continuation of $\delta_{0}$ to $\mathbb{R}$ :

$$
\begin{aligned}
\delta_{0}(x)= & 1+\sum_{\ell=1}^{\infty} \frac{(-1)^{\ell}}{(\ell !)^{2}} \xi^{2 \ell}[1](x)+\sum_{\ell=0}^{\infty} \frac{(-1)^{\ell+1}}{\ell !(\ell+1) !} \xi^{2 \ell+1}[1](x)\left(\mathbf{e}^{+}-\mathbf{e}^{-}\right) \\
= & 1+\sum_{\ell=1}^{\infty} \frac{(-1)^{\ell}}{(\ell !)^{2}}\left(x^{2} \prod_{j=1}^{\ell-1}\left(x^{2}-j^{2}\right)+\ell x \prod_{j=1}^{\ell-1}\left(x^{2}-j^{2}\right)\left(\mathbf{e}^{+} \mathbf{e}^{-}-\mathbf{e}^{-} \mathbf{e}^{+}\right)\right) \\
& -\sum_{\ell=0}^{\infty} \frac{(-1)^{\ell+1}}{\ell !(\ell+1) !} x \prod_{j=1}^{\ell}\left(x^{2}-j^{2}\right)\left(\mathbf{e}^{+} \mathbf{e}^{-}-\mathbf{e}^{-} \mathbf{e}^{+}\right) \\
= & 1+\sum_{\ell=1}^{\infty} \frac{(-1)^{\ell}}{(\ell !)^{2}} x^{2} \prod_{j=1}^{\ell-1}\left(x^{2}-j^{2}\right)
\end{aligned}
$$

We prove that this function converges for all $x \in \mathbb{R}$ to $\frac{\sin (\pi x)}{\pi x}$, which can then be regarded as the continuous expansion of the discrete delta function. We first show that the series (19) equals $\prod_{j=1}^{\infty}\left(1-\frac{x^{2}}{j^{2}}\right)$. We then invoke the Weierstrass factorization theorem in complex analysis, which asserts that an entire function can be represented by a product involving its zeroes. A corollary of the Weierstrass factorization theorem states that $\frac{\sin (\pi x)}{\pi x}=\prod_{j=1}^{\infty}\left(1-\frac{x^{2}}{j^{2}}\right), \forall x \in \mathbb{R}$, thus ending the proof. We show, by induction on $N$, that

$$
\delta_{0}(x)=\prod_{j=1}^{N}\left(1-\frac{x^{2}}{j^{2}}\right)+\sum_{\ell=N+1}^{\infty} \frac{(-1)^{\ell}}{\ell !^{2}} x^{2} \prod_{j=1}^{\ell-1}\left(x^{2}-j^{2}\right) .
$$

Hence, we first take $N=1,2$ :

$$
\begin{aligned}
\delta_{0}(x) & =1-x^{2}+\sum_{\ell=2}^{\infty} \frac{(-1)^{\ell}}{(\ell !)^{2}} x^{2} \prod_{j=1}^{\ell-1}\left(x^{2}-j^{2}\right) \\
\delta_{0}(x) & =1-x^{2}+\frac{1}{4} x^{2}\left(x^{2}-1\right)+\sum_{\ell=3}^{\infty} \frac{(-1)^{\ell}}{(\ell !)^{2}} x^{2} \prod_{j=1}^{\ell-1}\left(x^{2}-j^{2}\right) \\
& =\left(1-x^{2}\right)\left(1-\frac{x^{2}}{4}\right)+\sum_{\ell=3}^{\infty} \frac{(-1)^{\ell}}{(\ell !)^{2}} x^{2} \prod_{j=1}^{\ell-1}\left(x^{2}-j^{2}\right)
\end{aligned}
$$

Now assume (induction hypothesis) that for all $N \leqslant k-1$ the statement (20) holds. We then 
choose $k$ and consider

$$
\begin{aligned}
\delta_{0}(x) & =\prod_{j=1}^{k-1}\left(1-\frac{x^{2}}{j^{2}}\right)+\sum_{\ell=k}^{\infty} \frac{(-1)^{\ell}}{\ell !^{2}} x^{2} \prod_{j=1}^{\ell-1}\left(x^{2}-j^{2}\right) \\
& =\prod_{j=1}^{k-1}\left(1-\frac{x^{2}}{j^{2}}\right)+\frac{(-1)^{k}}{k !^{2}} x^{2} \prod_{j=1}^{k-1}\left(x^{2}-j^{2}\right)+\sum_{\ell=k+1}^{\infty} \frac{(-1)^{\ell}}{\ell !^{2}} x^{2} \prod_{j=1}^{\ell-1}\left(x^{2}-j^{2}\right) \\
& =\prod_{j=1}^{k-1}\left(1-\frac{x^{2}}{j^{2}}\right)-\frac{x^{2}}{k !^{2}} \prod_{j=1}^{k-1} j^{2}\left(1-\frac{x^{2}}{j^{2}}\right)+\sum_{\ell=k+1}^{\infty} \frac{(-1)^{\ell}}{\ell !^{2}} x^{2} \prod_{j=1}^{\ell-1}\left(x^{2}-j^{2}\right) \\
& =\prod_{j=1}^{k-1}\left(1-\frac{x^{2}}{j^{2}}\right)-\frac{x^{2}}{k^{2}} \prod_{j=1}^{k-1}\left(1-\frac{x^{2}}{j^{2}}\right)+\sum_{\ell=k+1}^{\infty} \frac{(-1)^{\ell}}{\ell !^{2}} x^{2} \prod_{j=1}^{\ell-1}\left(x^{2}-j^{2}\right) \\
& =\prod_{j=1}^{k}\left(1-\frac{x^{2}}{j^{2}}\right)+\sum_{\ell=k+1}^{\infty} \frac{(-1)^{\ell}}{\ell !^{2}} x^{2} \prod_{j=1}^{\ell-1}\left(x^{2}-j^{2}\right)
\end{aligned}
$$

which concludes the induction. Taking the limit $N \rightarrow \infty$ then shows

$$
\delta_{0}(x)=\prod_{j=1}^{\infty}\left(1-\frac{x^{2}}{j^{2}}\right)=\frac{\sin (\pi x)}{\pi x} .
$$

When we introduced the $\xi$ 's, we made the choice to let them satisfy $\xi^{k}[1](0)=0$, for all $k$. Thus, when we consider all continuous functions (defined on $\mathbb{R}$ ) which have the same function values in the integers, we have made a choice for one of these functions to be considered special. It would thus follow that the "special" continuous function which, on the integers, has the same values as the discrete delta function, would be the sinc function $\frac{\sin (\pi x)}{\pi x}$. Sampling a continuous function $f$ thus is nothing else then restricting $f$ to the grid, decomposing it into discrete delta functions and subsequently expanding it again to the whole of $\mathbb{R}$ :

$$
f(x)=\sum_{n=-\infty}^{\infty} f(n) \frac{\sin (\pi(x-n))}{\pi(x-n)}
$$

Remark 6.1. When we consider the discrete delta function on a general grid $\mathbb{Z}_{h}^{m}$ with mesh width $h>0$ :

$$
\delta_{0}(x)= \begin{cases}1, & x=0 \\ 0, & x \neq 0,\end{cases}
$$

its discrete Taylor series expansion is dependent on the mesh width $h$ :

$$
\begin{aligned}
\delta_{0} & =\sum_{\ell=0}^{\infty} \frac{(-1)^{\ell}}{h^{2 \ell} \ell !^{2}} \xi^{2 \ell}[1]+\sum_{\ell=0}^{\infty} \frac{(-1)^{\ell+1}}{h^{2 \ell+1} \ell !(\ell+1) !} \xi^{2 \ell+1}[1]\left(\mathbf{e}^{+}-\mathbf{e}^{-}\right) \\
& =\sum_{\ell=0}^{\infty} \frac{(-1)^{\ell}}{h^{2 \ell} \ell !^{2}} x^{2} \prod_{j=1}^{\ell-1}\left(x^{2}-(j h)^{2}\right)=\frac{\sin \left(\pi \frac{x}{h}\right)}{\pi \frac{x}{h}}
\end{aligned}
$$

Decomposing a discrete function $f$ into discrete delta functions

$$
f(x)=\sum_{j=-\infty}^{\infty} f(j h) \delta_{j h}(x)=\sum_{j=-\infty}^{\infty} f(j h) \frac{\sin \left(\pi\left(\frac{x}{h}-j\right)\right)}{\pi\left(\frac{x}{h}-j\right)}
$$

which shows the connection with the Shannon sampling theorem for $W=\frac{1}{2 h}$. 


\section{Conclusions}

In this paper, we established a Taylor series expansion theory for discrete functions. We considered convergence properties of the discrete Taylor series expansion and examined the equivalence of this Taylor series expansion and the original function. As an example, we applied the theory of the discrete Taylor series expansion to the discrete delta function in one dimension and showed the link with Shannon sampling.

\section{Acknowledgements}

The first author acknowledges support by the institutional grant no. B/10675/02 of Ghent University (BOF).

\section{References}

[1] F. Brackx, R. Delanghe, F. Sommen, Clifford Analysis, Research Notes in Mathematics 76, Pitman, London, 1982.

[2] K. Gürlebeck, A. Hommel, On finite difference potentials and their applications in a discrete function theory, Math. Meth. Appl. Sci. 25, 2002, pp. 1563-1576.

[3] F. Brackx, H. De Schepper, F. Sommen, L. Van de Voorde, Discrete Clifford analysis: an overview, Cubo 11(1), 2009, pp. 55-71.

[4] F. Brackx, H. De Schepper, F. Sommen, L. Van de Voorde, Discrete Clifford analysis: a germ of function theory. In: I. Sabadini, M. Shapiro, F. Sommen (eds.), Hypercomplex Analysis, Birkhäuser, 2009, pp. 37-53.

[5] H. De Ridder, H. De Schepper, U. Kähler, F. Sommen, Discrete function theory based on skew Weyl relations, Proc. Amer. Math. Soc. 138, 2010, pp. 3241-3256.

[6] H. De Ridder, H. De Schepper, F. Sommen, The Cauchy-Kovalevskaya Extension Theorem in Discrete Clifford Analysis, Comm. Pure Appl. Math 10(4), 2011, pp. 1097 - 1109.

[7] H. De Ridder, H. De Schepper, F. Sommen, Fueter polynomials in discrete Clifford analysis, Math. Zeit. 272(1-2), 2012, pp. 253-268.

[8] N. Faustino, U. Kähler, Fischer decomposition for difference Dirac operators, Adv. Appl. Cliff. Alg. 17(1), 2007, pp. 37-58.

[9] N. Faustino, Discrete Clifford analysis, Ph.D. thesis, Universidade de Aveiro, Aveiro, 2009.

[10] N. Faustino, U. Kähler, F. Sommen, Discrete Dirac operators in Clifford analysis, Adv. Appl. Cliff. Alg. 17(3), 2007, pp. 451-467.

[11] C. E. Shannon, A mathematical theory of communication, Bell. Sys. Tech. J., vol. 27, 1948, $379-423,623-56$.

[12] C. E. Shannon, Communication in the presence of noise, Proc. IRE, vol. 37, 1949, pp. 10-21. 


\section{A Technical lemmas}

The first lemma concentrates on one fixed dimension.

Lemma A.1. Let $f$ be a discrete function on $\mathbb{Z}_{h}^{m}$. Assume that for a given $j \in\{1, \ldots, m\}, a$ given $k \in \mathbb{N}$ and a given point $a \in \mathbb{Z}_{h}^{m}$, it holds that

$$
\partial_{j}^{r} f(a)=0, \quad \text { for all } r=0, \ldots, 2 k
$$

Then

$$
f\left(a+s h \mathbf{e}_{j}\right)=0, \quad \text { for all } s=0, \ldots, k
$$

We aim to establish that if two discrete functions have "enough" equal finite differences in a point, they must be equivalent. To prove this statement we however first state an auxiliary lemma which shows exactly the equality of which finite differences leads to the equivalence of which function values.

Lemma A.2. Let $x=\left(x_{1}, \ldots, x_{m}\right)$ be an arbitrary, but fixed point, and consider the $m$-dimensional rectangle centered at the origin, which has $x$ as one of its corner points. Now assume that the discrete function $f$ takes the value zero in all points of this $m$-dimensional rectangle, apart from the $2^{m}$ corner points, i.e. $f$ is assumed to be zero on the set

$$
\left\{y \in \mathbb{Z}_{h}^{m}:\left|y_{i}\right| \leqslant\left|x_{i}\right|, \forall i=1, \ldots, m\right\} \backslash\left\{y \in \mathbb{Z}_{h}^{m}:\left|y_{i}\right|=\left|x_{i}\right|, \forall i=1, \ldots, m\right\}
$$

If moreover

$$
\partial_{1}^{\alpha_{1}} \ldots \partial_{m}^{\alpha_{m}} f(0)=0, \forall \text { possible combinations with } \alpha_{i} \in\left\{2 \frac{\left|x_{i}\right|}{h}-1,2 \frac{\left|x_{i}\right|}{h}\right\}, \forall i=1, \ldots, m
$$

then it holds that the function $f$ is automatically zero in the corner points as well, i.e.

$$
f(y)=0, \forall y \in \mathbb{Z}_{h}^{m} \text { with }\left|y_{i}\right|=\left|x_{i}\right|, \forall i=1, \ldots, m
$$

Proof. Take $1 \leq r \leq m$ fixed and choose $\left\{\ell_{1}, \ldots, \ell_{r}\right\} \subset\{1, \ldots, m\}$. With this choice, we associate a point $c \in \mathbb{Z}_{h}^{r}$ for which each co-ordinate $c_{i}$ either equals $x_{\ell_{i}}$ or $-x_{\ell_{i}}, i=1, \ldots, r$. We will then prove, by induction on $r$ (where however $r \leq m$ ), that for all choices of $\left\{\ell_{1}, \ldots, \ell_{r}\right\} \subseteq\{1, \ldots, m\}$ and all choices of the corresponding point $c$, it holds that

$$
\sum_{\substack{y \in \mathbb{Z}_{h}^{m}:\left|y_{i}\right|=\left|x_{i}\right|, i=1, \ldots, m \\\left(y_{\ell_{1}}, \ldots, y_{\ell_{r}}\right)=c}} f(y)=0
$$

where the above sum thus is taken over all remaining corner points $y$ for which the co-ordinates $y_{\ell_{1}}, \ldots, y_{\ell_{r}}$ are fixed according to those of the chosen point $c$. Taking then in particular $r=m$, the above sum reduces to one single term, corresponding to the function value in one particular corner point $y$, which coincides with $c$. Since the equality holds for any choice of that corner point, this would then imply that the function value in all corner points indeed equals zero.

Hence, we first take $r=1$. In this case we will first consider condition (21) with $\alpha_{i}=2 \frac{\left|x_{i}\right|}{h}$, $\forall i=1, \ldots, m$, implying that

$$
0=\partial_{1}^{2 \frac{\left|x_{1}\right|}{h}} \ldots \partial_{m}^{2 \frac{\left|x_{m}\right|}{h}} f(0)=\sum_{y \in \mathbb{Z}_{h}^{m}:\left|y_{i}\right|=\left|x_{i}\right|, i=1, \ldots, m} f(y)
$$


since all other function values occurring in the involved finite differences are assumed to be zero. Next, we again consider (21), now taking for one fixed, but arbitrary $j \in\{1, \ldots, m\}$ the corresponding exponent $\alpha_{j}=2 \frac{\left|x_{j}\right|}{h}-1$, which yields

$$
0=\partial_{1}^{2 \frac{\left|x_{1}\right|}{h}} \ldots \partial_{j}^{2 \frac{\left|x_{j}\right|}{h}-1} \ldots \partial_{m}^{2 \frac{\left|x_{m}\right|}{h}} f(0)=\mathbf{e}_{j}^{+} \sum_{\substack{y:\left|y_{i}\right|=\left|x_{i}\right| \\ y_{j}=x_{j}}} f(y)-\mathbf{e}_{j}^{-} \sum_{\substack{y:\left|y_{i}\right|=\left|x_{i}\right| \\ y_{j}=-x_{j}}} f(y)
$$

where we have used an obvious abbreviated notation for the set $\left\{y \in \mathbb{Z}_{h}^{m}:\left|y_{i}\right|=\left|x_{i}\right|, i=1, \ldots, m\right\}$. Substitution of (23) herein gives

$$
0=\mathbf{e}_{j}^{+} \sum_{\substack{y:\left|y_{i}\right|=\left|x_{i}\right| \\ y_{j}=x_{j}}} f(y)-\mathbf{e}_{j}^{-} \sum_{\substack{| \\y:| y_{i}|=| x_{i} \mid \\ y_{j}=-x_{j}}} f(y) \stackrel{(23)}{=} \mathbf{e}_{j} \sum_{\substack{y:\left|y_{i}\right|=\left|x_{i}\right| \\ y_{j}=x_{j}}} f(y)
$$

eventually resulting into

$$
\sum_{\substack{\left|y_{i}\right|=\left|x_{i}\right| \\ y_{j}=x_{j}}} f(y)=\sum_{\substack{y:\left|y_{i}\right|=\left|x_{i}\right| \\ y_{j}=-x_{j}}} f(y)=0, \quad j=1, \ldots, m
$$

so that the statement holds for $r=1$.

Now assume (induction hypothesis) that for all choices of $\left\{\ell_{1}, \ldots, \ell_{r}\right\} \subset\{1, \ldots, m\}$ with $r \leqslant k-1$ and all choices of the corresponding point $c$ the statement (22) holds. We then choose $\left\{\ell_{1}, \ldots, \ell_{k}\right\} \subseteq$ $\{1, \ldots, m\}$ (induction step), and we consider condition (21) with $\alpha_{i}=2 \frac{\left|x_{i}\right|}{h}$ if $i \notin\left\{\ell_{1}, \ldots, \ell_{k}\right\}$ and $\alpha_{i}=2 \frac{\left|x_{i}\right|}{h}-1$ if $i \in\left\{\ell_{1}, \ldots, \ell_{k}\right\}$, resulting into

$$
0=\sum_{\text {all choices of } c} \sum_{\substack{y:\left|y_{i}\right|=\left|x_{i}\right| \\\left(y \ell_{1}, \ldots, y \ell_{k}\right)=c}}\left( \pm \mathbf{e}_{\ell_{1}}^{ \pm}\right) \ldots\left( \pm \mathbf{e}_{\ell_{k}}^{ \pm}\right) f(y)
$$

where the basis vector $\mathbf{e}_{\ell_{j}}^{+}$occurs whenever $c_{j}=x_{\ell_{j}}$, while $\mathbf{e}_{\ell_{j}}^{-}$occurs whenever $c_{j}=-x_{\ell_{j}}$, $j=1, \ldots, k$, and each basis vector is preceded by a plus or a minus sign accordingly. From Lemma A.3 below, we see that this indeed implies the statement for $r=k$, which completes the proof by induction.

While completing the induction step, we used the following auxiliary result.

Lemma A.3. Let $r \leqslant k-1$, let $\left\{\ell_{1}, \ldots, \ell_{r}\right\} \subseteq\{1, \ldots, m\}$, let $c$ be a point in $\mathbb{Z}_{h}^{r}$ for which each co-ordinate $c_{i}$ either equals $x_{\ell_{i}}$ or $-x_{\ell_{i}}, i=1, \ldots, r$, and assume that for all choices of $\left\{\ell_{1}, \ldots, \ell_{r}\right\} \subseteq\{1, \ldots, m\}$ and all choices of the point $c$, it holds that

$$
\sum_{\substack{y:\left|y_{i}\right|=\left|x_{i}\right| \\\left(y_{\ell_{1}}, \ldots, y_{\ell_{r}}\right)=c}} f(y)=0
$$

Next, take $1 \leq p \leq k$, and correspondingly, $\left\{\ell_{1}, \ldots, \ell_{p}\right\} \subseteq\{1, \ldots, m\}$ and a point $c \in \mathbb{Z}_{h}^{p}$. Furthermore, put $s=k-p$, let $\left\{j_{1}, \ldots, j_{s}\right\} \subseteq\{1, \ldots, m\} \backslash\left\{\ell_{1}, \ldots, \ell_{p}\right\}$ and let $c^{\prime}$ be a point in $\mathbb{Z}_{h}^{s}$ for which each co-ordinate $c_{i}^{\prime}$ either equals $x_{j_{i}}$ or $-x_{j_{i}}, i=1, \ldots, s$. Then, if for all choices of $\left\{\ell_{1}, \ldots, \ell_{p}\right\}$, for all choices of $\left\{j_{1}, \ldots, j_{s}\right\}$ and for all choices of the point $c^{\prime}$, it holds that

$$
\sum_{\text {all choices of } c} \sum_{\substack{y:\left|y_{i}\right|=\left|x_{i}\right| \\\left(y_{\ell_{1}}, \ldots, y_{\ell_{p}}\right)=c \\\left(y_{j_{1}}, \ldots, y_{j_{s}}\right)=c^{\prime}}}\left( \pm \mathbf{e}_{\ell_{1}}^{ \pm}\right) \ldots\left( \pm \mathbf{e}_{\ell_{p}}^{ \pm}\right) f(y)=0
$$


(where the plus and minus signs are taken as explained in the proof of Lemma A.2), this implies that for all choices of the point $c$ it holds that

$$
\sum_{\begin{array}{c}
y:\left|y_{i}\right|=\left|x_{i}\right| \\
\left(y_{\ell_{1}}, \ldots, y_{\ell_{p}}\right)=c \\
\left(y_{j_{1}}, \ldots, y_{j_{s}}\right)=c^{\prime}
\end{array}} f(y)=0
$$

We now may formulate an immediate corollary of Lemma A.2, where we consider finite differences in a general point $a$ instead of in the origin.

Corollary A.1. Let $a=\left(a_{1}, \ldots, a_{m}\right)$ and $x=\left(x_{1}, \ldots, x_{m}\right)$ be arbitrary, but fixed points, and consider the m-dimensional rectangle centered at a, having $x$ as one of its corner points. Now assume that $f$ takes the value zero in all points of this $m$-dimensional rectangle, apart from the $2^{m}$ corner points. If moreover

$$
\partial_{1}^{\alpha_{1}} \ldots \partial_{m}^{\alpha_{m}} f(a)=0, \quad \text { for all } \alpha_{i} \in\left\{2 \frac{\left|x_{i}-a_{i}\right|}{h}-1,2 \frac{\left|x_{i}-a_{i}\right|}{h}\right\}, i=1, \ldots, m
$$

then $f$ automatically is zero in all corner points as well.

Example A.1. We demonstrate for the standard grid $(h=1)$ in two dimensions how lemmas A.1 and A.2 lead to the proof of theorem 3.1.

- Step one: $f(\underline{0})=0$

- Step two: from lemma A.1 we see that if $\partial_{j} f(\underline{0})=\partial_{j}^{2} f(\underline{0})=0$, for each $j=1,2$, then

$$
f(1,0)=f(-1,0)=f(0,1)=f(0,-1)=0
$$

- Step three: we apply lemma A.2 with $\underline{x}=(1,1): f$ is zero on

$$
\left\{\left(y_{1}, y_{2}\right) \in \mathbb{Z}^{2}|| y_{i}|\leqslant| x_{i} \mid, i=1,2\right\} \backslash\{(1,1),(1,-1),(-1,1),(-1,-1)\}
$$

so if $\partial_{1} \partial_{2} f(\underline{0})=\partial_{1}^{2} \partial_{2} f(\underline{0})=\partial_{1} \partial_{2}^{2} f(\underline{0})=\partial_{1}^{2} \partial_{2}^{2} f(\underline{0})=0$, then lemma A.2 implies that

$$
f(1,1)=f(-1,1)=f(-1,1)=f(-1,-1)=0
$$

- Step four: from lemma A.1 we see that if furthermore $\partial_{j}^{3} f(\underline{0})=\partial_{j}^{4} f(\underline{0})=0, j=1,2$, then

$$
f(2,0)=f(-2,0)=f(0,2)=f(0,-2)=0
$$

- Step five. We apply lemma A.2 two times: once with $\underline{x}=(1,2)$ and once with $\underline{x}=(2,1)$. If

$$
\begin{aligned}
& \partial_{1} \partial_{2}^{3} f(\underline{0})=\partial_{1} \partial_{2}^{4} f(\underline{0})=\partial_{1}^{2} \partial_{2}^{3} f(\underline{0})=\partial_{1}^{2} \partial_{2}^{4} f(\underline{0})=0 \\
& \partial_{1}^{3} \partial_{2} f(\underline{0})=\partial_{1}^{4} \partial_{2} f(\underline{0})=\partial_{1}^{3} \partial_{2}^{2} f(\underline{0})=\partial_{1}^{4} \partial_{2}^{2} f(\underline{0})=0
\end{aligned}
$$

then lemma A.2 implies that

$$
\begin{aligned}
& f(1,2)=f(1,-2)=f(-1,2)=f(-1,-2)=0 \\
& f(2,1)=f(2,-1)=f(-2,1)=f(-2,-1)=0
\end{aligned}
$$



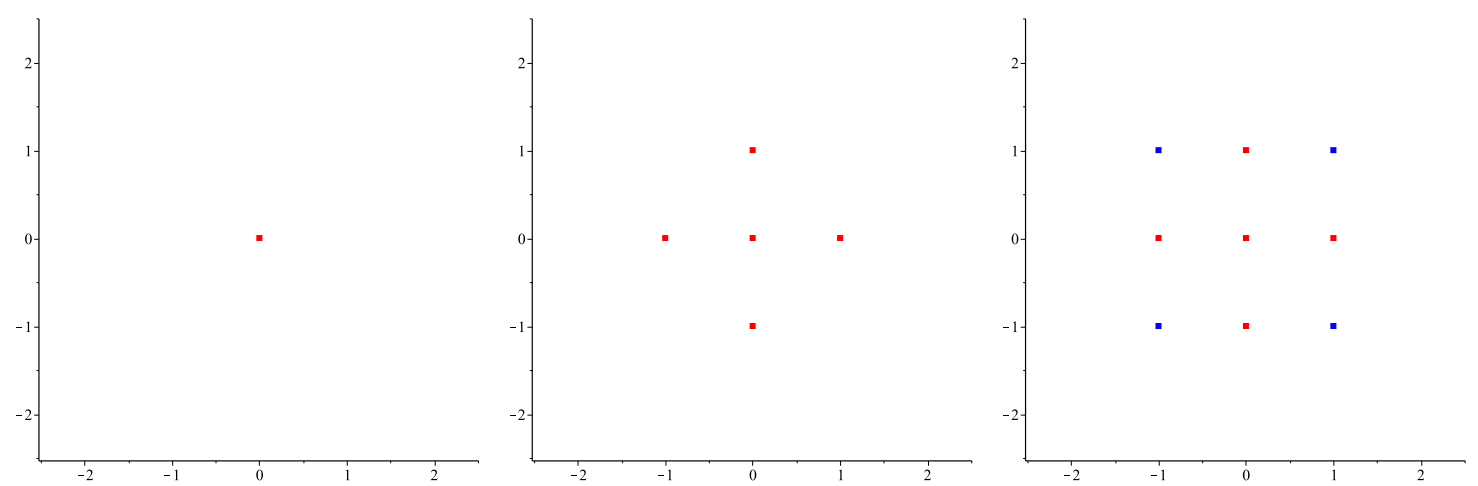

Figure 1: Step 1 (left), step 2 (midden) and step 3 (right).
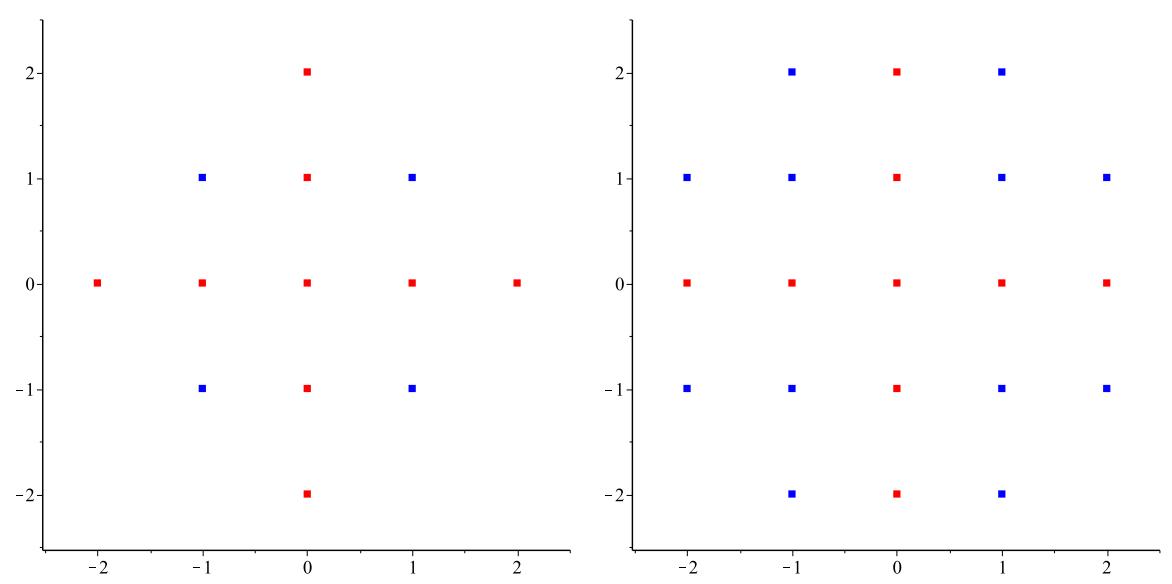

Figure 2: Step 4 (left) and step 5 (right). 